\title{
An Adaptive Method for Fully Automatic Liver Segmentation in Medical MRI-Images
}

\author{
Roaa G. Mohamed \\ Faculty of Computer \& \\ Info. Sciences \\ Ain Shams University, \\ Cairo 11566, Egypt
}

\author{
Noha A. Seada \\ Faculty of Computer \& \\ Info. Sciences \\ Ain Shams University, \\ Cairo 11566, Egypt
}

\author{
Salma Hamdy \\ Faculty of Computer \& \\ Info. Sciences \\ Ain Shams University, \\ Cairo 11566, Egypt
}

\author{
Mostafa G. M. \\ Mostafa \\ Faculty of Computer \& \\ Info. Sciences \\ Ain Shams University, \\ Cairo 11566, Egypt
}

\begin{abstract}
Despite the importance of the liver segmentation in the medical images for efficient noninvasive diagnosis, few studies found in the literatures for fully automated methods for liver segmentation in Magnetic Resonance Imaging (MRI) compared to that in Computed Tomography (CT) scans. Motivated by this, we propose an adaptive fully automatic liver segmentation method for MRI images based on thresholding and Bayesian classification. Bayesian classifications have proved to be highly robust to various image degradations. It only requires a small amount of training data to estimate the parameters necessary for classification, which is a huge advantage in medical applications. Furthermore, the Bayesian model is robust when large uncertainties are involved in medical image analysis problems. The proposed method is successfully tested on many MRI cases acquired from different patients, in various sizes. Experiments proved the robustness of the proposed liver automatic segmentation process even on data from different scanner types. The segmentation accuracy of the model has a mean Dice Similarity Coefficient (DSC) of $95.5 \%$ for MRI datasets.
\end{abstract}

\section{General Terms}

Image processing, Computer Vision.

\section{Keywords}

Medical Image Analysis, Automatic Liver Segmentation, Bayesian Classifier.

\section{INTRODUCTION}

Liver segmentation is a key condition and a major step in the diagnosis of liver diseases and surgical planning, whether the diagnosis or treatments involve surgical ways (surgeries) or non-surgical ways (medical imaging). Surgical intervention is one of the ultimate treatment options and surgical planning is considered a major step in successful surgical operations. For example, tumor removal planning includes quantification of the tumor size, its location, and trajectories during surgery, in addition to the effect of tumor removal on other neighboring organs, and the success rate of the surgery. Medical imaging is considered a non-surgical and painless way in clinical practice for the diagnosis of some liver diseases [1][2].

Thus, accurate liver segmentation has many benefits in different medical aspects. It has a direct application on planning, monitoring, and treatment of different types of pathologies such as hepatocellular carcinoma or cirrhosis diseases. And due to the high accuracy needed, segmentation of the liver is often done on images with high spatial resolution, i.e., Computed Tomography (CT) or Magnetic Resonance Images (MRI).

Currently, fully automatic liver segmentation in medical images remains an unsolved problem [3]. This is mainly due to reasons concerned with medical imaging in general, and reasons related to the liver in particular as Figure 1 shows. Pictures of livers show irregularity in the liver shape, size and appearance, across patients as in (a and e), in addition to liver sharing similar intensity distributions with its surrounding organs. For as it is known, the liver is a soft organ with variable shape. This makes it more challenging especially for automatic liver detection. Furthermore, many infections, presence of tumors, or other abnormalities have a strong effect on the appearance, volume and the shape of the liver as in Fig. 1(d). Hence, most of the time, clearly defined edges are not visible on many sides of the liver [4]-[6].

As for medical imaging technology, such as CT and MRI, abdominal images are represented in gray level rather than color. These are often very noisy that inevitably, ambiguous boundaries appear between the liver and its adjacent organs, including abdominal wall, right kidney, heart, stomach and gallbladder. Sometimes, due to poor quality of imaging, the boundaries completely disappear, and segmentation becomes very difficult as in Figure 1(b). This raise an urgent need for a more efficient and accurate volume segmentation technique [7]-[10]. Although liver volume can be calculated using some common formulas such as Mosteller formula depending on age, weight and height of the patient, these formulas are not accurate in the case of pathological livers [11].

Many researchers address liver segmentation because of the importance of the subject. However, there is no global technique that can be used with all kinds of images; each technique has its own strengths and weaknesses. Interested readers can refer to [2], [12], [13] for a thorough survey of liver segmentation techniques. Also, quantitative segmentation validation methods are considered among the open issues in the literature. Many validation methods, including area overlap, Dice measure, and Hausdroff distances [14]-[16], are usually used in addition to the visual validation of the cases by an expert, which is still a necessary measure for the validity of the segmentation method.

Bayesian classifier has gained significant attention in the scientific community for solving computer vision tasks such as object recognition, classification and segmentation[17], often out-competing state-of-the art methods. It only requires a small amount of training data to estimate the parameters necessary for classification, and strategies have proved this classifier to be highly robust to varying image appearance. 
Various statistical methods have been used for classifying medical images in the field of diseases diagnosis. Some of these methods require prior assumptions and are less capable of dealing with massive and complicated nonlinear and dependent data. In general, images classification methods are categorized into supervised (and unsupervised models.
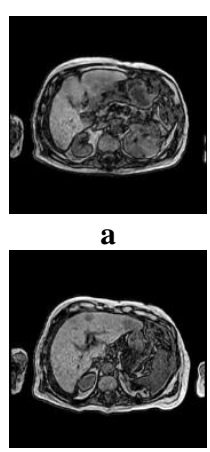

d

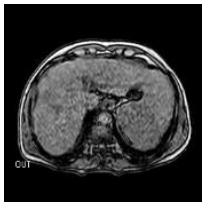

b

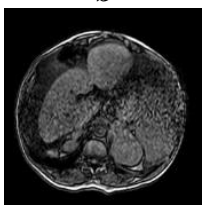

e

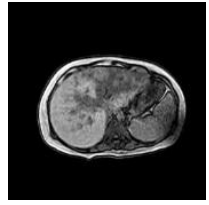

c
Figure 1: Some difficulties of liver segmentation irregularity in the liver shape, size and appearance, liver sharing similar intensity distributions with its surrounding organs, presence of tumors, or other abnormalities have a strong effect on the appearance, volume and the shape of the liver, and ambiguous boundaries appear between the liver and its adjacent organs.

Bayesian classification represents a supervised learning method [18], [19] as well as a statistical method for classification. This method assumes an underlying probabilistic model and allows capturing uncertainty about the model in a principled way by determining probabilities of the outcomes. It can solve diagnostic and predictive problems. In addition, Bayesian model is robust when large uncertainties are involved in medical image analysis problems [20].

In this work, we propose a model for liver segmentation from MRI images based on Bayesian classification. We validate our method using both careful visual examination by an expert radiologist, and Dice Similarity Coefficient (DSC) [21] measure for calculating error and accuracy.

The rest of the paper is organized as follows: Section 2 presents a survey of related work on liver segmentation. Section 3 introduces the data set, mathematical model and proposed segmentation method. Section 4 describes the experiments conducted to test the method and discusses the results. Finally, conclusions are presented in section 5.

\section{RELATED WORK}

Liver segmentation methods can be classified into three classes [22] in line with the level of required human interaction. These are automatic methods that require no user interaction, semi-automatic methods that require some initial user interaction, and interactive methods (manual) that require full user interaction from the beginning to end of segmentation. Manual segmentation is a time intensive and tedious task as it involves working with large amount of image slices and datasets. Also, the segmentation results rely on the experience and skills of the radiologists and because of inconsistencies of liver images mentioned earlier, the liver boundaries may be identified differently by different radiologists and even by the exact same radiologist at another time. Therefore, several automatic and semi-automatic liver segmentation methods from medical images have been proposed to help overcome problems of manual segmentation.
Chen and Foruzan in [23] presented a graph-cut based method to segment the liver from MR images. K-means clustering was used to automatically obtain the object (liver) and background (non-liver) seeds (regions) in every slice of the volume without user interaction.

Freiman et al. [24] proposed a new Bayesian probabilistic level set framework for automatic liver segmentation and liver volume calculation from CT images. Their proposed unified framework employs an edge indication function and a novel nonlinear variable force, which is based on probabilistic distribution at the pixel level and is used to adjust the level set evolution. As for the liver volume estimation, they used a multi-resolution iterative scheme that repeatedly applied smoothed Bayesian classification to identify the liver and other organs.

Cheng et al. [25] proposed a level set approach for liver segmentation from abdomen MRI series images, where shape prior knowledge is combined with the improved Chan-Vese's model. The proposed approach can overcome the leakage and over-segmentation problems.

Linguraru et al [26] presented an automated segmentation of livers from abdominal CT images, in which an affine invariant shape parameterization is combined with a geodesic active contour and graph cuts. A geodesic active contour locally corrects the segmentations of organs in abnormal images of abnormal liver, while the optimized graph cuts segment the vasculature and hepatic tumors using shape and enhancement constraints.

Lim et al. [27] proposed an approach for automatic liver segmentation from CT images for volume liver estimation, where they analyze the intensity distribution of CT samples to obtain a priori model to determine the coherent regions of the liver. A morphological filter is recursively applied with region-labeling and clustering to detect the search range and to generate the initial liver boundary, which then leads to liver volume estimation.

Seo in [28] proposed a method to hepatic tumor segmentation from CT images using composite hypotheses. In the beginning, histogram transformation, multimodal threshold, maximum a posteriori decision, and binary morphological filtering, are used to segment the liver. For finding an optimal threshold the hepatic vessels are removed from the liver. The optimal threshold value is then utilized with minimum total probability error to segment a hepatic tumor. The results show that the proposed method is very useful for diagnosis of the normal and abnormal liver, although tested on 10 cases only.

Rikxoort et al. [29] proposed a method that starts with preprocessing steps to determine the vertical scan range of the liver and to rotate the scan so that the subject is in supine position. Then voxel labeling with K-nearest-neighbor is performed, and a final smoothing filter is applied to obtain a fine segmentation. The method is tested on only 10 test scans.

Platero and Tobar in [30] presented a combination between the probabilistic atlas and multi atlas segmentation for liver segmentation from CT images. It is based on minimizing a discrete energy function using graph cuts. Applications often focus as well on segmenting the vessel and tumor. By using graph cuts method which can be easily achieved since, when segmenting the vessel or tumor, the "background" liver is homogeneous.

No evaluation or comparison can be made of the various works to segment the liver from medical images because of 
the fact that every author utilizes a small private dataset and different performance measure criteria. For an objective comparison, for the most part, knowledge performance measures are required. Each existing method has its own advantages and disadvantages, and none is fully robust. From our review of the previous proposed researches, it is concluded that there is still need for a robust and efficient method for automatic liver segmentation.

\section{THE PROPOSED METHOD}

Among the different classification approaches that are based on statistical models is the Bayesian classification. It is one of the most effective and efficient algorithms and has been successfully applied to many medical problems. Studies show that Bayesian classification is well suited for medical application and has high performance rate in most of the examined medical problems [31].

Bayesian classification provides several practical learning algorithms such as Naive Bayes, Gaussian Naive Bayes, Multinomial Naive Bayes, Averaged One-Dependence Estimators (AODE), Bayesian Belief Network (BBN), and Bayesian Network (BN) [32]. It provides a useful perspective for understanding and evaluating many learning algorithms where prior knowledge and observed data can be combined. The method calculates explicit probabilities for hypotheses and it is robust to noisy input data [33].

Bayesian classification is a pixel-wise operator. It classifies each pixel in the image using statistical models for the classes from the training data. It is based on the following formula [27] [37]:

$$
p\left(c_{i} \mid x\right)=\frac{p\left(x \mid c_{i}\right) \cdot p\left(c_{i}\right)}{p(x)},
$$

where $p\left(c_{i} \mid x\right)$, the posterior, is the probability of instance $x$ being in class $c_{i}$, this is what we are trying to compute, $p\left(x \mid c_{i}\right)$, the likelihood; is the probability of generating instance $\mathrm{x}$ given class $c_{i}, p\left(c_{i}\right)$, the prior, is the probability of occurrence of class $c_{i}$, this is just how frequent the class $c_{i}$ is in the image, and $p(x)$, the evidence, is the probability of occurrence of instance $x$, which can be ignored, since it is a normalization factor and is the same for all classes. That is, to compute the posterior, we only need to calculate $p\left(c_{1} \mid x\right)$ and $p\left(c_{2} \mid x\right)$ for the feature $\mathrm{x}$.

We use the Gaussian model for modeling the data, that is, we use

$$
p\left(x \mid c_{i}\right)=N\left(\mu_{i}, \sigma_{i}^{2}\right)
$$

Where:

$$
N\left(\mu_{i}, \sigma_{i}^{2}\right)=\frac{1}{\sqrt{2 \pi \sigma_{i}^{2}}} e^{-\frac{\left(x-\mu_{i}\right)^{2}}{2 \sigma_{i}^{2}}}
$$

where $\mu$ is the mean and $\sigma$ is the standard deviation.

Ideally, for automatic liver segmentation from MRI images, the scanned liver tissue would have some degree of consistency to distinguish it from the surrounding tissues. A pixel of a certain value is classified as a liver pixel as opposed to a non-liver or background pixel. However, as mentioned earlier, quality issues may prevent such clear distinction and pixels end up incorrectly segmented. We build a Bayesian model to classify every pixel of the input image as belonging to the liver tissue or not based on its probability of being a liver pixel or not. The probabilities are pre-calculated from a set of labeled training images and used to decide if a pixel in a new test case is liver. A pseudo code of the algorithm for the proposed method is shown in Fig. 2.

\begin{tabular}{l} 
Algorithm 1: Adaptive Fully Automatic Liver \\
Segmentation \\
\hline Input: A dataset of image volume of abdominal MRI \\
images. \\
Output: Segmented liver images in the dataset. \\
for each image in the datasets do \\
Select the middle slice from the image volume. \\
Apply thresholding and select the largest connected \\
component as the liver. \\
Compute the parameters $\mu$ and $\sigma$ for liver class and other \\
classes in the image (non-liver and background). \\
for each pixel in each image in the dataset do \\
Compute the likelihoods for each class using Eq. (2). \\
Compute the posteriors of each class using Eq. (1). \\
Label the pixel with the class that have the largest \\
posterior. \\
end for \\
Apply post processing procedures for extracting the liver \\
(considering size and location). \\
end for
\end{tabular}

Figure 2: Pseudocode for the proposed method.

In our method, we label the training data to categorize three classes: liver, non-liver, and background of the image, as $\mathrm{C} 1$, $\mathrm{C} 2$ and $\mathrm{C} 3$, respectively. The training data is obtained using the method we proposed in a previous research [34] . Fig. 3 shows a sample of the training data for each class.

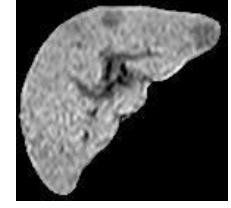

a. Liver (Class1)

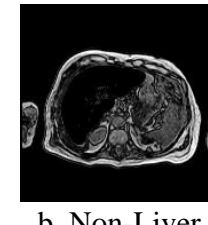

b. Non-Liver (Class2)



(Class3)
Figure 3. Training data.

\section{RESULTS AND DISCUSSIONS}

It should be stated that there are no benchmark datasets for abdomen MRI images available to test the proposed methods of liver segmentation. Furthermore, none of the authors of previous works have made their own datasets public. Since the developed algorithm is meant to be capable of segmenting of MRI images available in clinics, we collected datasets from several medical centers and hospitals; where the MRI images are all of type T1; a type preferred by radiologists for its quality [4]. All abdominal MRI datasets have been acquired of the liver. The cases are from 20 patients, ranging in age between 7 and 70 years old; 3 of which have normal livers, and the rest have diseased livers.

The proposed algorithm is designed for automatic segmentation of the liver. It has achieved promising results on the collected dataset of T1 images. Some test cases are shown in Figure 4. The Dice Similarity Coefficient (DSC) [21] is used to evaluate the segmentation accuracy. The algorithm achieved a DSC of $95.5 \%$ for the test cases. Figure 5 shows a sample of our results against manual annotation. 


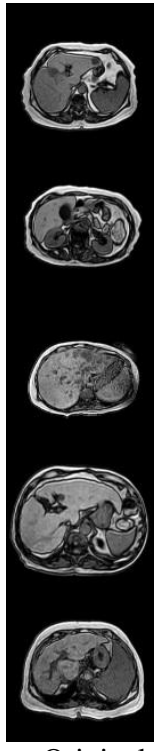

a. Original image.

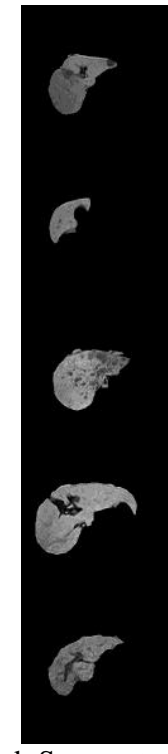

b.Segmented liver.

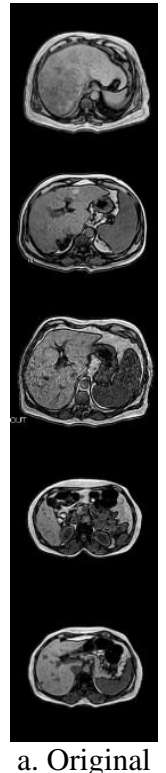

image.

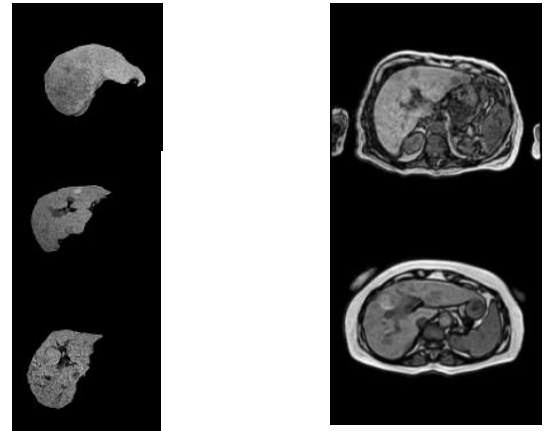

a. original image



b. liver segmented before postprocessing

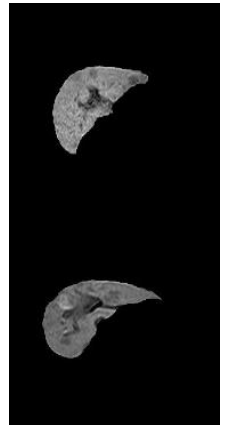

c. liver segmented after postprocessing
Figure 5: Example of post processing step

Ideally, the algorithm would be assessed against previous similar techniques. But unfortunately, an objective comparison among different systems would not be accurate and fair, due to the lack of a common dataset with gold standards, i.e. a commonly accepted manual segmentation, and a unique measure of discrepancy between the automatic and the manual segmentation of each abdominal organ. Besides, most authors employ their own private datasets.

However, since the same data set was used in both proposed methods (this Bayesian model and our previous work in [37]), a comparison of the two seems equitable, and is carried out in terms of their respective segmentation results, accuracy, and speed of completing the automatic segmentation function. Sample results from both techniques are shown in Table 1.

It is noted that the proposed Bayesian method is faster than the method from [27]. This is because the latter's reliance on active contour technique, which depends on a certain number of frequencies to obtain good results. The average speed rate of liver segmentation with active contours was 0.96 seconds, while the average rate of segmentation speed of the Bayesian model reached 0.22 seconds. The segmentation accuracy for both methods was close, with active contour reaching $95 \%$, while the Bayesian model scoring slightly higher with $95.5 \%$.

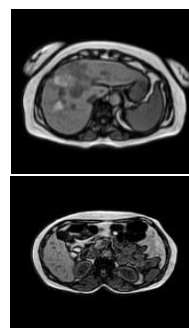

a.
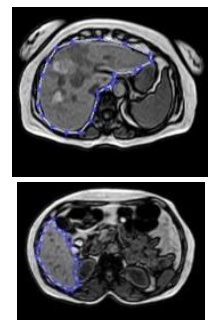

b.

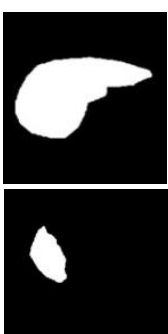

c.

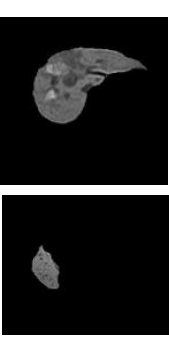

d.
Figure 5. a. Original MRI slice b. Manual annotation with DSC c. Manual segmentation d. Automatic segmentation. 


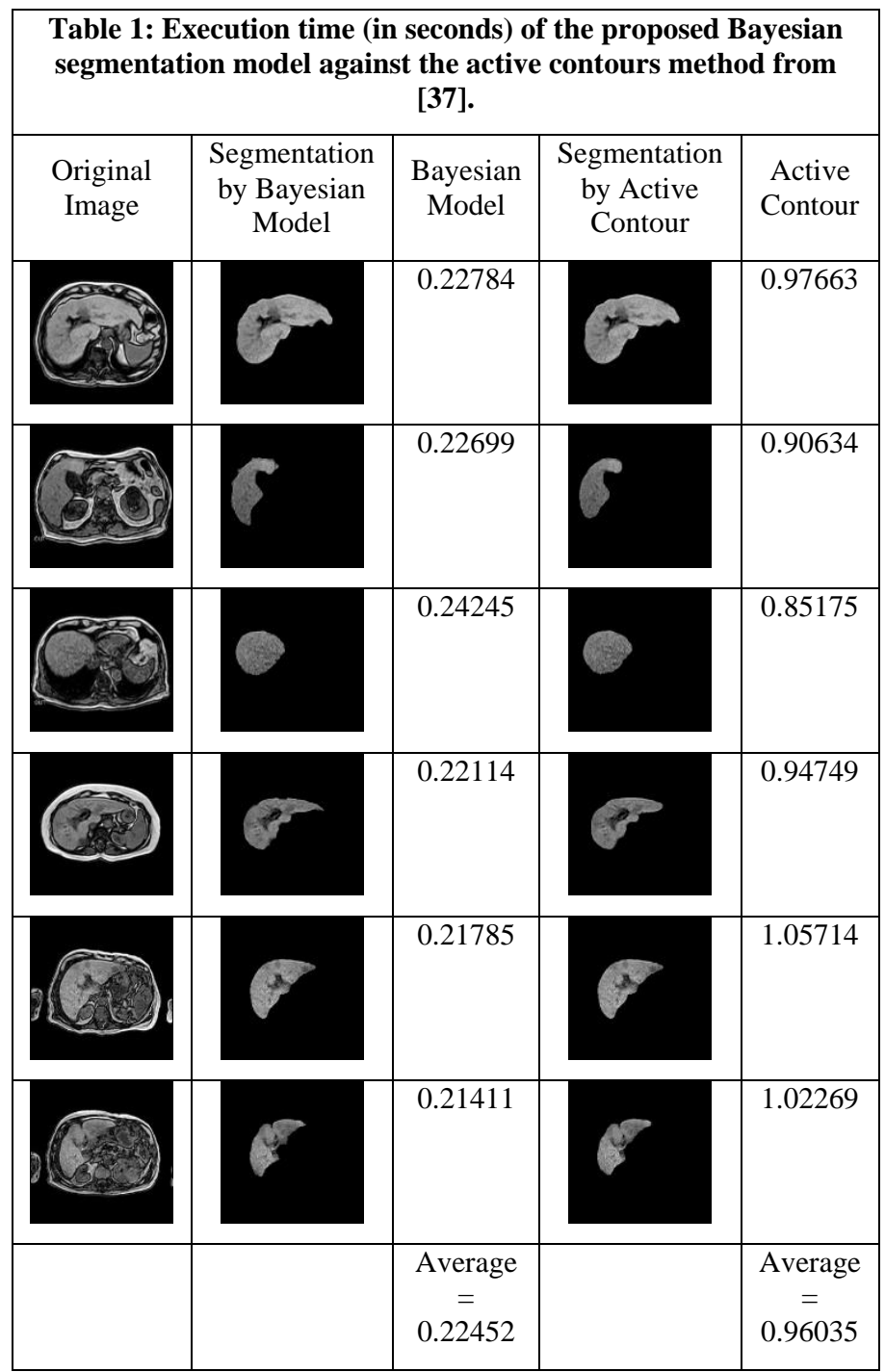

\section{CONCLUSION}

A good liver segmentation is the first important step in liver treatment planning; this paper presents a method for automatically liver segmentation. The proposed method, which is based on Bayesian classification, automatically extracts the liver from MRI scans. It utilizes adaptive parameters according to the characteristics of a particular patient (the new slice) to accommodate patient-specific liver features during segmentation. For as it is known, the usage of common parameters for all patients decreases the efficiency of the segmentation. The method has been tested on T1 MRI imaging datasets from different scanners. Experiments demonstrated the liver successful segmented from the datasets with accuracy having a mean Dice Similarity Coefficient (DSC) of $95.5 \%$. The average processing time for the segmentation was about 0.22 seconds. The time and accuracy attained proves the reliability of the proposed algorithm. Moreover, the novelty of the approach lies in its full automation (no user interaction needed). Also, no preprocessing steps are needed to identify a region of interest or remove surrounding organs to easily detect the liver. Furthermore, MRI images in the datasets used for testing are for both normal and abnormal liver tissues, confirming that the proposed technique can be used further for diagnosing the lesions on the surface of abnormal liver tissue. In future work, the model can be developed to solve the leakage problem in some cases, where the liver contour is not clear due to hepatomegaly. Also, a further step would be to segment and classify tumors from the unhealthy livers.

\section{REFERENCES}

[1] S. Kiruthika and I. K. Raj, "Liver Extraction Using Histogram and Morphology,” IJRET, pp. 245-249, 2016.

[2] I. Singh, "A Study of Effective Segmentation Techniques for Liver Segmentation," IJARCET, vol. 4, no. 4, pp. 1661-1666, 2015.

[3] S. Editor, A. Gefen, and R. Aviv, " Soft Tissue Biomechanical Modeling for Computer Assisted Surgery," Springer,2012.

[4] A. Bereciartua, A. Picon, A. Galdran, and P. Iriondo, "Automatic 3D model-based method for liver segmentation in MRI based on active contours and total variation minimization," Biomed. Signal Process. Control, vol. 20, pp. 71-77, 2015.

[5] Y. Chen, Z. Wang, J. Hu, W. Zhao, and Q. Wu, "The domain knowledge based graph-cut model for liver CT segmentation," Biomed. Signal Process. Control, vol. 7, no. 6, pp. 591-598, 2012.

[6] N. Aldeek, R. S. Alomari, M. B. Al-Zoubi, and H. Hiary, 
"Liver segmentation from abdomen CT images with bayesian model," J. Theor. Appl. Inf. Technol., vol. 60, no. 3, pp. 483-490, 2014.

[7] D. Chi, Y. Zhao, and M. Li, "Automatic liver MR image segmentation with self-organizing map and hierarchical agglomerative clustering method," Proc. - 2010 3rd Int. Congr. Image Signal Process. CISP 2010, vol. 3, pp. 1333-1337, 2010.

[8] Wolf, I., van Rikxoort, E., Raicu, D.S., Rau, A.M., Nemeth, G., Meinzer, H., Li, S., Li, R., Lennon, B., Lee, J., Lange, T., Lamecker, H., Rousson, M., Rusko, L., Wimmer, A., Waite, J., Susomboon, R., Soza, G., Sorantin, E., Slagmolen, P., Shimizu, A., Seghers, D., Schmidt, G., Saddi, K., Kobatake, H., Kitney, R., Kainmueller, D., Bello, F., Bekes, G., Beichel, R., Becker, C., Beck, A., Bauer, C., Aurich, V., Arzhaeva, Y., Styner, M., van Ginneken, B., Binnig, G., Bischof, H., Hornegger, J., Grenacher, L., Furukawa, D., Furst, J., Fidrich, M., Dawant, B., Cordova, A., Chi, Y., Cashman, P., Bornik, A., Heimann, T, "Comparison and evaluation of methods for liver segmentation from CT datasets," IEEE Trans. Med. Imaging, vol. 28, no. 8, pp. 12511265, 2009.

[9] H. Masoumi, A. Behrad, M. A. Pourmina, and A. Roosta, "Automatic liver segmentation in MRI images using an iterative watershed algorithm and artificial neural network," Biomed. Signal Process. Control, vol. 7, no. 5, pp. 429-437, 2012.

[10] A. Rafiee, H. Masoumi, and A. Roosta, "Using neural network for liver detection in abdominal MRI images," ICSIPA09 - 2009 IEEE Int. Conf. Signal Image Process. Appl. Conf. Proc., pp. 21-26, 2009.

[11] M. Moghbel, S. Mashohor, R. Mahmud, and M. I. Bin, "Automatic Liver Segmentation on Computed Tomography Using Random Walkers for Treatment Planning," EXCLI, no. 2003, pp. 500-517, 2016.

[12] N. Khan and I. Ahmed, "Overview of Technical Elements of Liver Segmentation," IJACSA, vol. 7, no. 12, pp. 271-278, 2016.

[13] S. Luo, X. Li, and J. Li, "Review on the Methods of Automatic Liver Segmentation from Abdominal Images," J. Comput. Commun., vol. 2, no. 2, pp. 1-7, 2014.

[14] S. S. Kumar, R. S. Moni, and J. Rajeesh, "Automatic Segmentation of Liver and Tumor for CAD of Liver," Advances, vol. 2, no. 1, pp. 63-70, 2011.

[15] K. H. Zou, W. M. Wells, R. Kikinis, and S. K. Warfield, "Three validation metrics for automated probabilistic image segmentation of brain tumours," Stat. Med., vol. 23, no. 8, pp. 1259-1282, 2004.

[16] A. Hann et al., "Algorithm guided outlining of 105 pancreatic cancer liver metastases in Ultrasound," Sci. Rep., vol. 7, p. 12779, 2017.

[17] J. A. Mangai, J. Nayak, and V. S. Kumar, "A Novel Approach for Classifying Medical Images Using Data Mining Techniques," Int. J. Comput. Sci. Electron. Eng., vol. 1, no. 2, 2013.

[18] R.Mallika, "Fraud Detection using Supervised Learning Algorithms," Ijarcce, vol. 6, no. 6, pp. 6-10, 2017.
[19] S.Amidwar, "Plagiarism Detection Using Supervised Machine Learning," JETIR, vol. 4, no. June, 2017.

[20] R. Blanco, I. Inza, M. Merino, J. Quiroga, and P. Larrañaga, "Feature selection in Bayesian classifiers for the prognosis of survival of cirrhotic patients treated with TIPS,” J. Biomed. Inform., vol. 38, no. 5, pp. 376-388, 2005.

[21] K. H. Zou, S. K. Warfield, A. Bharatha, M. R. Kaus, S. J. Haker, W. M. Wells III, F. A. Jolesz, and R. Kikinis, "Statistical Validation of Image Segmentation Quality Based on a Spatial Overlap Index," Acad. Radiol., vol 11, no. 2, pp. 178-189, 2004.

[22] A. M. Mharib, A. R. Ramli, S. Mashohor, and R. B. Mahmood, "Survey on liver CT image segmentation methods," Artif. Intell. Rev., vol. 37, no. 2, pp. 83-95, 2012.

[23] Y. W. Chen, K. Tsubokawa, and A. H. Foruzan, "Liver Segmentation from Low Contrast Open MR Scans Using K-Means Clustering and Graph-Cuts," vol. 54, no. February, 2016.

[24] M. Freiman, O. Eliassaf, Y. Taieb, L. Joskowicz, Y. Azraq, and J. Sosna, "An iterative Bayesian approach for nearly automatic liver segmentation: Algorithm and validation,” Int. J. Comput. Assist. Radiol. Surg., vol. 3, no. 5, pp. 439-446, 2008.

[25] K. Cheng, L. Gu, and J. Xu, "A Novel Shape Prior Based Level Set Method for Liver Segmentation From MR Images," no. x, pp. 144-147, 2008.

[26] M. G. Linguraru, W. J. Richbourg, J. M. Watt, V. Pamulapati, and R. M. Summers, "Liver and tumor segmentation and analysis from CT of diseased patients via a generic affine invariant shape parameterization and graph cuts," Lect. Notes Comput. Sci. (including Subser. Lect. Notes Artif. Intell. Lect. Notes Bioinformatics), vol. 7029 LNCS, pp. 198-206, 2012.

[27] S. J. Lim, Y. Y. Jeong, and Y. S. Ho, "Automatic liver segmentation for volume measurement in CT Images," J. Vis. Commun. Image Represent., vol. 17, no. 4, pp. 860$875,2006$.

[28] K. Seo, "Automatic hepatic tumor segmentation using composite hypotheses," Lect. notes Comput. Sci., vol. 3656, p. 922, 2005.

[29] E. Van Rikxoort, Y. Arzhaeva, and B. Van Ginneken, "Automatic segmentation of the liver in computed tomography scans with voxel classification and atlas matching," Proc. MICCAI ..., no. September, pp. 101-108, 2007.

[30] C. Platero and M. C. Tobar, "A multiatlas segmentation using graph cuts with applications to liver segmentation in CT scans," Comput. Math. Methods Med., vol. 2014, 2014.

[31] R. T. Ribeiro, R. T. Marinho, and J. Miguel Sanches, "Classification and staging of chronic liver disease from multimodal data," IEEE Trans. Biomed. Eng., vol. 60, no. 5, pp. 1336-1344, 2013.

[32] J. Li, C. Zhang, T. Wang, and Y. Zhang, "Generalized additive Bayesian network classifiers," Proc. Int. Jt. Conf. Artif. ..., no. x, pp. 913-918, 2007.

[33] M. Ceci, "Naive bayesian learning from structural 
data," pp. 23-27, 2005.

[34] L. Li, "Bayesian Classification and Regression With High Dimensional Features," 2007.

[35] R. V. de Schoot, D. Kaplan, J. Denissen, J. B. Asendorpf, F. J. Neyer, and M. A. G. van Aken, "A Gentle Introduction to Bayesian Analysis: Applications to Developmental Research," Child Dev., vol. 85, no. 3, pp. 842-860, 2014.
[36] M. Samsó, M. J. Palumbo, M. Radermacher, J. S. Liu, and C. E. Lawrence, "A Bayesian method for classification of images from electron micrographs," J. Struct. Biol., vol. 138, no. 3, pp. 157-170, 2002.

[37] R.G.Mohamed, N. A. Seada, S.Hamdy, M.G.M.Mostafa, "Automatic Liver Segmentation from Abdominal MRI Images Using Active Contours." IJCA, vol.176,no $\quad 1, \quad$ October 2017. 